\title{
Cross-Lingual Named Entity Recognition via Wikification
}

\author{
Chen-Tse Tsai, Stephen Mayhew, and Dan Roth \\ University of Illinois, Urbana-Champaign \\ 201 N. Goodwin, Urbana, Illinois, 61801 \\ \{ctsai12, mayhew2, danr\}aillinois.edu
}

\begin{abstract}
Named Entity Recognition (NER) models for language $L$ are typically trained using annotated data in that language. We study cross-lingual NER, where a model for NER in $L$ is trained on another, source, language (or multiple source languages). We introduce a language independent method for NER, building on cross-lingual wikification, a technique that grounds words and phrases in nonEnglish text into English Wikipedia entries. Thus, mentions in any language can be described using a set of categories and FreeBase types, yielding, as we show, strong language-independent features. With this insight, we propose an NER model that can be applied to all languages in Wikipedia. When trained on English, our model outperforms comparable approaches on the standard CoNLL datasets (Spanish, German, and Dutch) and also performs very well on lowresource languages (e.g., Turkish, Taga$\log$, Yoruba, Bengali, and Tamil) that have significantly smaller Wikipedia. Moreover, our method allows us to train on multiple source languages, typically improving NER results on the target languages. Finally, we show that our languageindependent features can be used also to enhance monolingual NER systems, yielding improved results for all 9 languages.
\end{abstract}

\section{Introduction}

Named Entity Recognition (NER) is the task of identifying and typing phrases that contain the names of persons, organizations, locations, and so on. It is an information extraction task that is im- portant for understanding large bodies of text and is considered an essential pre-processing stage in Natural Language Processing (NLP) and Information Retrieval systems.

NER is successful for languages which have a large amount of annotated data, but for languages with little to no annotated data, this task becomes very challenging. There are two common approaches to address the lack of training data problem. The first approach is to automatically generate annotated training data in the target language from Wikipedia articles or from parallel corpora. The performance of this method depends on the quality of the generated data and how well the language-specific features are explored. The second approach is to train a model on another language which has abundant training data, and then apply the model directly on test documents in the target language. This direct transfer technique relies on developing language-independent features. Note that these two approaches are orthogonal and can be used together.

In this paper, we focus on the second, direct transfer setting. We propose a cross-lingual NER model which is trained on annotated documents in one or multiple source languages, and can be applied to all languages in Wikipedia. The model depends on a cross-lingual wikifier, which only requires multilingual Wikipedia, no sentencealigned or word-aligned parallel text is needed.

The key contribution of this paper is the development of a method that makes use of crosslingual wikification and entity linking (Tsai and Roth, 2016; Ji et al., 2015; Ji et al., 2016; Moro et al., 2014) to generate language-independent features for NER, and showing how useful this can be for training NER models with no annotation in the target language. Given a mention (sub-string) from a document written in a foreign language, the goal of cross-lingual wikification is to find the cor- 


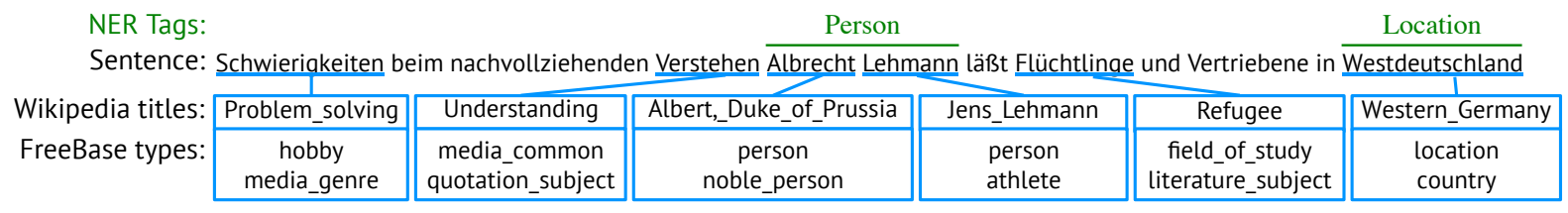

Figure 1: An example of a German sentence. We ground each word to the English Wikipedia using a cross-lingual wikifier. A word is not linked if it is a stop word or the wikifier returns NIL. We can see that the FreeBase types are strong signals to NER even with imperfect disambiguation.

responding title in the English Wikipedia. Traditionally, wikification has been considered a downstream task of NER. That is, a named entity recognizer is first applied to identify mentions of interest, and then a wikifier is used to ground the extracted mentions to Wikipedia entries. In contrast to this traditional pipeline, we show that the ability to ground and disambiguate words is very useful to NER. By grounding every $n$-gram to the English Wikipedia, we obtain useful clues to NER, regardless of the target language.

Figure 1 shows an example of a German sentence. We use a cross-lingual wikifier to ground each word to the English Wikipedia. We can see that even though the disambiguation is not perfect, the FreeBase types still provide valuable information. That is, although "Albrecht Lehmann" is not an entry in Wikipedia, the wikifier still links "Albrecht" and "Lehmann" to people. Since words in any language are grounded to the English Wikipedia, the corresponding Wikipedia categories and Freebase types can be used as language-independent features.

The proposed model significantly outperforms comparable direct transfer methods on the Spanish, Dutch, and German CoNLL data. We also evaluate the model on five low-resource languages: Turkish, Tagalog, Yoruba, Bengali, and Tamil. Due to small sizes of Wikipedia, the overall performance is not as good as the CoNLL experiments. Nevertheless, the wikifier features still give significant improvements, and the proposed direct transfer model outperforms the state of the art, which assumes parallel text and some interaction with a native speaker of the target language. In addition, we show that the proposed languageindependent features not only perform well on the direct transfer scenario, but also improve monolingual models, which are trained on the target language. Another advantage of the proposed direct transfer model is that we can train on documents from multiple languages together, and further im- prove the results.

\section{Related Work}

There are three main branches of work for extending NLP systems to many languages: projection across parallel data, Wikipedia-based approaches, and direct transfer. Projection and direct transfer take advantage of the success of NLP tools on high-resource languages. Wikipediabased approaches exploit the fact that, by editing Wikipedia, thousands of people have made annotations in hundreds of languages.

\subsection{Projection}

Projection methods take a parallel corpus between source and target languages, annotate the source side, and push annotations across learned alignment edges. Assuming that source side annotations are of high quality, success depends largely on the quality of the alignments, which depends, in turn, on the size of the parallel data, and the difficulty of aligning with the target language.

There is work on projection for POS tagging (Yarowsky et al., 2001; Das and Petrov, 2011; Duong et al., 2014), NER (Wang and Manning, 2014; Kim et al., 2012; Ehrmann et al., 2011), and parsing (Hwa et al., 2005; McDonald et al., 2011).

Wang and Manning (2014) show that projecting expectations of labels instead of hard labels can improve results. They experiment in two different settings: weakly-supervised, where only parallel data is available, and semi-supervised, where annotated training data is available along with unlabeled parallel data.

\subsection{Using Wikipedia}

Wikipedia has been used for a large number of NLP tasks, from use as a semantic space (Gabrilovich and Markovitch, 2007; Chang et al., 2008; Song and Roth, 2014), to generating parallel data (Smith et al., 2010), to use in open information extraction (Wu and Weld, 2010). It has 
also been used to extract training data for NER, under the intuition that Wikipedia is already (partially) annotated with NER labels, in the form of links to pages. Nothman et al. (2012) generate silver-standard NER data from Wikipedia using link targets, and other heuristics. This can be gathered for any language in Wikipedia, but several of the heuristics depend on language-specific rules. Al-Rfou et al. (2015) generate training data from Wikipedia articles using a similar manner. The polyglot word embeddings (Al-Rfou et al., 2013) are used as features in their NER model. Although the features are delexicalized, the embeddings are unique to each language, and so the model cannot transfer.

Kim et al. (2012) use Wikipedia to generate parallel sentences with NE annotations. They propose a semi-CRF model for aligning entities in parallel sentences. Results are very strong on Wikipedia data. This is a hybrid approach in that it is supervised projection using Wikipedia.

Our work is most closely related to Kazama and Torisawa (2007). They do NER using Wikipedia category features for each mention. However, their method for wikifying text is not robust to ambiguity, and they only do monolingual NER.

Sil and Yates (2013) create a joint model for NER and entity linking in English. They avoid the traditional pipeline by overgenerating mentions in the first stage and using NER features to rank candidates. While the results are promising, the model is not scalable to other languages because it requires both a trained NER and a NP chunker.

\subsection{Direct Transfer}

In direct transfer once trains a model in a highresource setting using delexicalized features, that is, features that do not depend on word forms, and then directly applies it to text in a new language.

Täckström et al. (2012) experimented with direct transfer of dependency parsing and NER, and showed that using word cluster features can help, especially if the clusters are forced to conform across languages. The cross-lingual word clusters were induced using large parallel corpora.

Building on this work, Täckström (2012) focuses solely on NER, and includes experiments on self-training and multi-source transfer for NER. Their experiments are orthogonal to ours, and could be combined nicely. This work is closest to ours in terms of method, and therefore we com-

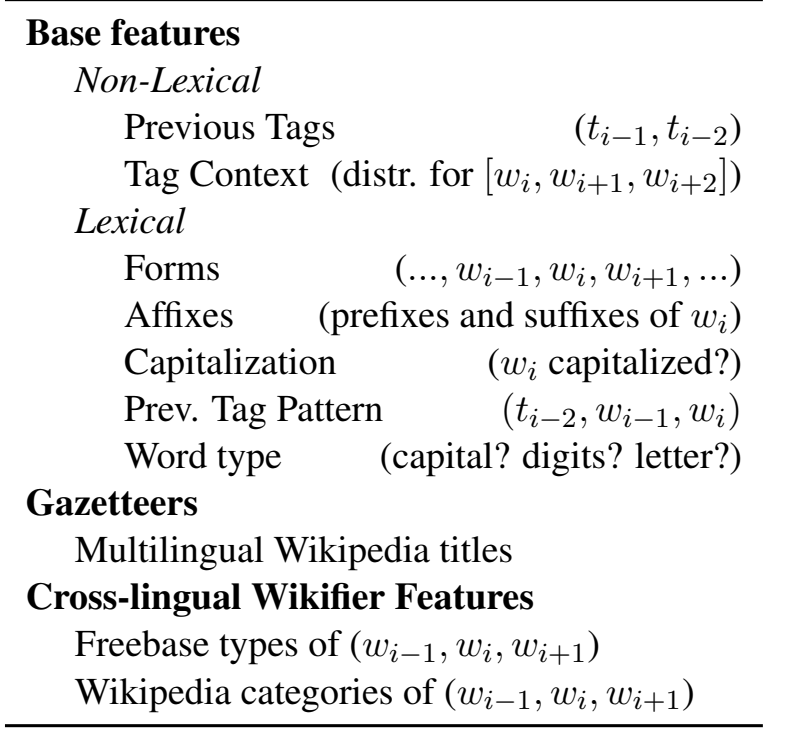

Table 1: Feature groups. Base features are the features used by Ratinov and Roth (2009), the state of the art English NER model. Gazetteers and crosslingual wikifier features are described in detail in Section 3.

pare against it in our experiments.

Our work falls under the umbrella of direct transfer methods combined with the use of Wikipedia. We introduce wikifier features, which are truly delexicalized, and use Wikipedia as a source of information for each language.

\section{Named Entity Recognition Model}

We use the state of the art English NER model of Ratinov and Roth (2009) as the base model. This model approaches NER as a multiclass classification problem with greedy decoding, using the BIO labeling scheme. The underlying classifier is averaged perceptron.

Table 1 summarizes the features used in our model. These can be divided into a base set of standard features which are included in Ratinov and Roth (2009), a set of gazetteer features which are based on titles in multilingual Wikipedia, and our novel cross-lingual wikifier features. The base set of features can be further divided into nonlexical and lexical categories.

\subsection{Base Features}

Non-Lexical Features Ratinov and Roth (2009) uses a small number of non-lexical features. For example, the previous tag feature is useful in predicting I- tags, because the previous tag should never be an $\mathrm{O}$. The tag context feature looks in a 
1000 word history and gathers statistics over tags assigned to words $\left[w_{i}, w_{i+1}, w_{i+2}\right]$. These features are included in all experiments.

In contrast with (Täckström et al., 2012), we do not use POS tags as features. We could not get the universal POS tags for all languages in our experiments, and an earlier experiment indicated that adding POS tags does not improve the performance due to the accuracy of tagger.

Lexical Features Lexical features are very important for monolingual NER. In the direct transfer setting, lexical features are useful if the target language is close to the training language. We use a small number of simple features, including word forms, affixes, capitalization, and tag patterns. The latter feature considers a small window (at most 2 tokens) before the word in question. If there is a named entity in the window, it makes a feature out of NETag $+w_{i-2}+w_{i-1}$. Word type features simply indicate whether the word in question is all capitalized, is all digits, or is all letters.

\subsection{Gazetteer Features}

One of the larger performance improvements in Ratinov and Roth (2009) came from the use of (partial matches with) gazetteers. We include gazetteers also in our model, except we gather them in each language from Wikipedia. As in Ratinov and Roth (2009), we use the gazetteers as features. Specifically, we group them by topic, and use the name of the gazetteer file as the feature.

The method iteratively extends a short window to the right of the word in question. As the window increases in size, we search all gazetteers for occurrences of the phrase in the window. If we find a match, we add a feature to each word in the phrase according to its position in the phrase, either B for beginning, or I for inside.

This method generalizes gazetteers to unseen entities. For example, given the phrase "Bill and Melinda Gates Foundation", "Bill" is marked as both B-PersonNames and B-Organizations, while "Foundation" is marked as I-Organizations. Imagine encountering at test time a fictional organization called "Dave and Sue Harris Foundation." Although there is no gazetteer that contains this name, we have learned that "B-PersonName and B-PersonName B-PersonName Foundation" is a strong signal for an organization.

\subsection{Cross-lingual Wikifier Features}

As shown in Figure 1, disambiguating words to Wikipedia entries allows us to obtain useful information for NER from the corresponding FreeBase types and Wikipedia categories. A crosslingual wikifier grounds words and phrases of non-English languages to the English Wikipedia, which provides language-independent features for transferring an NER model directly.

We use the system proposed in Tsai and Roth (2016), which grounds input strings to the intersection of (the title spaces of) the English and the target language Wikipedias. The only requirement is a multilingual Wikipedia dump and it can be applied to all languages in Wikipedia.

Since we want to ground every $n$-gram $(n \leq 4)$ in the document, deviating from the normal usage that only considers a few mentions of interest, we modify the system in the following two ways:

- The original candidate generation process queries the index by both whole input string and the individual tokens of the string. For the $n$-grams where $n>1$, we generate title candidates only according to the whole string, not individual tokens. If we allow generating title candidates based on individual tokens then, for instance, the bigram "in Germany" will be linked to the title Germany thus wrongly considered as a named entity.

- The original ranking model includes the embeddings of other mentions in the document as features. It is clear that if we know what other important entities exist in the document, they provide useful clues to disambiguate a mention. However, if we want to wikify all $n$-grams, it makes no sense to include all of them as features, since the ranking model has already included features from TF-IDF weighted context words.

After wikifying every $n$-gram ${ }^{1}$, we set the types of each $n$-gram as the coarse- and fine-grained FreeBase types and Wikipedia categories from the top 2 title candidates returned by wikifier. For each word $w_{i}$, we use the types of $w_{i}, w_{i+1}$, and $w_{i-1}$, and the types of the $n$-grams which contain $w_{i}$ as features. Moreover, we also include wikifier's ranking features from the top candidate as features. This could serve as a linker (Ratinov et

\footnotetext{
${ }^{1}$ We set $n$ to 4 in all our experiments.
} 
al., 2011), which rejects the top prediction if it has a low confidence.

\section{Experiments and Analysis}

In this section, we conduct experiments to validate and analyze the proposed NER model. First, we show that adding wikifier features improves results on monolingual NER. Second, we show that wikifier features are strong signals in direct transfer of a trained NER model across languages. Finally, we explore the importance of Wikipedia size to the quality of wikifier features and study the use of multiple source languages.

\subsection{Datasets}

We use data from CoNLL2002/2003 shared tasks (Tjong Kim Sang, 2002; Tjong Kim Sang and De Meulder, 2003). The 4 languages represented are English, German, Spanish, and Dutch, each annotated using the IOB1 labeling scheme, which we convert to the BIO labeling scheme. All training is on the train set, and testing is on the test set. The evaluation metric for all experiments is phrase level F1, as explained in (Tjong Kim Sang, 2002). In order to experiment on a broader range of languages, we also use data from the REFLEX (Simpson et al., 2008) and LORELEI projects. From LORELEI, we use Turkish, ${ }^{2}$ From REFLEX, we use Bengali, Tagalog, Tamil, and Yoruba. ${ }^{3}$ While Turkish, Tagalog, and Yoruba each has a few non-Latin characters, Bengali and Tamil are with an entirely non-Latin script. This is a major reason for inclusion in our experiments. We use the same set of test documents as used in Zhang et al. (2016). All other documents in the REFLEX and LORELEI packages are used as the training documents in our monolingual experiments. We refer to these five languages collectively as low-resource languages.

Besides PER, LOC, and ORG, some lowresource languages contain TIME tags and TTL tags, which represented titles in text, such as Secretary, President, or Minister. Since such words are not tagged in the CoNLL training data, we opted to simply remove these tags. On the other hand, there is no MISC tag in the low-resource languages. Instead, many MISC-tagged entities in the CoNLL datasets have LOC tags in the REFLEX and LORELEI packages, e.g., Italian and

\footnotetext{
${ }^{2}$ LDC2014E115

${ }^{3}$ LDC2015E13,LDC2015E90,LDC2015E83,LDC2015E91
}

Chinese. We modify a MISC-tagged word to LOC tag if it is grounded to an entity with location as a FreeBase type, and remove all the other MISC tags in the training data. This process of changing MISC tags is only done when we train on CoNLL documents and test on low-resource languages.

The only requirement to build the cross-lingual wikifier model is a multilingual Wikipedia dump, and it can be trivially applied to all languages in Wikipedia. The top section of Table 2 lists Wikipedia sizes in terms of articles, ${ }^{4}$ the number of titles linked to English titles, and the number of training and test mentions for each language.

Besides the English gazetteers used in Ratinov and Roth (2009), we collect gazetteers for each language using Wikipedia titles. A Wikipedia title is included in the list for person names if it contains FreeBase type person. Similarly, we also create a location list and an organization list for each language. The total number of names in the gazetteers of each language is listed in Table 2.

\subsection{Monolingual Experiments}

We begin by showing that wikifier features help when we train and test on the same language. The middle section of Table 2 shows these results.

In the 'Wikifier only' row, we use only wikifier features and previous tags features. This is intended to show the predictive power of wikifier features alone. Without using any lexical features, it gets good scores on the languages that have a large Wikipedia. These numbers represent the quality of the cross-lingual wikifier in that language, which in turn is correlated with the size of Wikipedia and size of the intersection with English Wikipedia.

The next row, 'Base features', shows that lexical features are always better than wikifier features only. This agrees with the common wisdom that lexical features are important for NER.

Adding gazetteers to the base features improves by more than 3 points for higher-resource languages. This is because the low-resource languages have much smaller gazetteers which have lower coverage than other languages' gazetteers.

Finally, the '+Wikifier' row shows that our proposed features are valuable even in combination with strong features. It improves upon base features and gazetteer features for all 9 languages.

\footnotetext{
${ }^{4}$ From https://en.wikipedia.org/wiki/ List_of_Wikipedias, retrieved March 2016
} 


\begin{tabular}{|c|c|c|c|c|c|c|c|c|c|c|}
\hline \multirow[b]{2}{*}{ APPROACH } & \multicolumn{7}{|c|}{ Latin Script } & \multicolumn{2}{|c|}{ Non-Latin Script } & \multirow[b]{2}{*}{ AVG } \\
\hline & EN & NL & $\mathrm{DE}$ & ES & TR & $\mathrm{TL}$ & YO & $\mathrm{BN}$ & $\mathrm{TA}$ & \\
\hline Wiki size & $5.1 \mathrm{M}$ & $1.9 \mathrm{M}$ & $1.9 \mathrm{M}$ & $1.3 \mathrm{M}$ & $269 \mathrm{~K}$ & $64 \mathrm{~K}$ & $31 \mathrm{~K}$ & $42 \mathrm{~K}$ & $85 \mathrm{~K}$ & - \\
\hline En. intersection & - & $755 \mathrm{~K}$ & $964 \mathrm{~K}$ & $757 \mathrm{~K}$ & $169 \mathrm{~K}$ & $49 \mathrm{~K}$ & $30 \mathrm{~K}$ & $34 \mathrm{~K}$ & $51 \mathrm{~K}$ & - \\
\hline Gazetteer size & $8.5 \mathrm{M}$ & $579 \mathrm{~K}$ & $1 \mathrm{M}$ & $943 \mathrm{~K}$ & $168 \mathrm{~K}$ & $54 \mathrm{~K}$ & $20 \mathrm{~K}$ & $29 \mathrm{~K}$ & $10 \mathrm{~K}$ & - \\
\hline Entities (train) & $23.5 \mathrm{~K}$ & $18.8 \mathrm{~K}$ & $11.9 \mathrm{~K}$ & $13.3 \mathrm{~K}$ & $5.1 \mathrm{~K}$ & $4.6 \mathrm{~K}$ & $4.1 \mathrm{~K}$ & $8.8 \mathrm{~K}$ & $7.0 \mathrm{~K}$ & - \\
\hline Entities (test) & $5.6 \mathrm{~K}$ & $3.6 \mathrm{~K}$ & $3.7 \mathrm{~K}$ & $3.9 \mathrm{~K}$ & $2.2 \mathrm{~K}$ & $3.4 \mathrm{~K}$ & $3.4 \mathrm{~K}$ & $3.5 \mathrm{~K}$ & $4.6 \mathrm{~K}$ & - \\
\hline \multicolumn{11}{|c|}{ Monolingual Experiments } \\
\hline Wikifier only & 71.57 & 57.02 & 49.74 & 60.13 & 52.84 & 51.02 & 29.35 & 47.78 & 38.05 & 50.83 \\
\hline Base Features & 85.50 & 76.64 & 65.88 & 80.66 & 64.98 & 75.03 & 55.26 & 69.26 & 55.93 & 69.90 \\
\hline +Gazetteers & 89.49 & 82.41 & 69.31 & 83.62 & 70.41 & 76.71 & 57.12 & 69.51 & 57.10 & 72.89 \\
\hline +Wikifier & 89.92 & 84.49 & 73.13 & 83.87 & 73.86 & 77.64 & $\mathbf{5 7 . 6 0}$ & 71.15 & 60.02 & 74.72 \\
\hline \multicolumn{11}{|c|}{ Direct Transfer Experiments } \\
\hline Wikifier only & & 40.44 & 39.83 & 43.82 & 41.79 & 42.11 & 27.91 & 43.27 & 29.64 & 38.01 \\
\hline Base Features & & 43.38 & 24.93 & 42.85 & 29.21 & 49.85 & 32.57 & 2.53 & 1.74 & 28.06 \\
\hline +Gazetteers & & 50.26 & 34.47 & 54.59 & 30.21 & 64.06 & 34.37 & 3.25 & 0.30 & 33.83 \\
\hline +Wikifier & & 61.56 & 48.12 & 60.55 & 47.12 & 65.44 & 36.65 & 18.18 & 5.65 & 41.41 \\
\hline \multirow{3}{*}{\multicolumn{2}{|c|}{$\begin{array}{l}\text { Täckström baseline } \\
\text { Täckström bitext clusters } \\
\text { Zhang et al. (2016) }\end{array}$}} & 48.4 & 23.5 & 45.6 & - & - & - & - & - & - \\
\hline & & 58.4 & 40.4 & 59.3 & - & - & - & - & - & - \\
\hline & & - & - & - & 43.6 & 51.3 & 36.0 & 34.8 & 26.0 & 38.3 \\
\hline
\end{tabular}

Table 2: Data sizes, monolingual experiments, and direct transfer experiments. Wiki size is the number of articles in Wikipedia. For monolingual experiments, we train the proposed model on the training data of the target languages. 'Wikifier only' uses the previous tags features also. For direct transfer experiments, all models are trained on CoNLL English training set. The rows marked Täckström come from (Täckström et al., 2012), and are the baseline and clustering result. The plus signs (+) signify cumulative addition. EN: English, NL: Dutch, DE: German, ES: Spanish, TR: Turkish, TL: Tagalog, YO: Yoruba, BN: Bengali, TA: Tamil.

These numbers may be less than state of the art because the features we use are designed for English, and may not capture lexical subtleties in every language. Nevertheless, they show that wikifier features have a non-trivial signal that has not been captured by other features.

\subsection{Direct Transfer Experiments}

We evaluate our direct transfer experiments by training on English and testing on the target language. The results from these experiments are shown in the bottom section of Table 2.

The 'Wikifier only' row shows that the wikifier features alone preserve a signal across languages. Interestingly, for both Bengali and Tamil, this is the strongest signal, and gets the highest score. If the lexical features are included when we train the English model, the learning algorithm will give them too much emphasis, thus decreasing the importance of the wikifier features. Since Bengali and Tamil use non-Latin scripts, no lexical feature in English will fire at test time. Thus, approaches that include base features perform poorly.

The results of 'Base features' can be viewed as a sort of language similarity to English, which, in this case, is related to lexical overlap and similarity between the scripts. Comparing to monolingual experiments, we can see that the lexical features become weak in the cross-lingual setting.

The gazetteer features are again shown to be very useful for almost all languages except Bengali and Tamil due to the reason explained in the monolingual experiment and to the inclusion of lexical features. For all other languages, the gain from adding gazetteers is even larger than it is in the monolingual setting.

For nearly every language, wikifier features help dramatically, which indicates that they are very good delexicalized features. Wikifier features add more than 10 points on Dutch, German, and Turkish.

The trend in Table 2 suggests the following strategy when we want to extract named entities in a new foreign language: It is better to include all features if the foreign language uses Latin script, since the names are likely to be mentioned similarly to the English names. Otherwise, using wikifier features only could be the best setting. 
Täckström et al. (2012) also directly transfer an English NER model using the same setting as ours: train on the CoNLL English training set and predict on the test set of other three languages. We compare our baseline transfer model (Base Features) to the row denoted by "Täckström baseline". Even though we do not use gold POS tags, we see that our results are comparable. The second Täckström row uses parallel text to induce multilingual word clustering. While this approach is orthogonal to ours, and could be used in tandem to get even better scores, we compare against it for lack of a more closely aligned scenario. We see that for each language, our approach significantly outperforms their approach.

We note that our numbers are comparable to those reported for WIKI-2 in Nothman et al. (2012) for the CoNLL languages (with the exception of German, where their result is higher). However, they require language-specific heuristics to generate silver-standard training data from Wikipedia articles. What they gain for single languages, they likely lose in generalization to other languages. This approach is orthogonal to ours; we, too, can use their silver-standard data in training.

For the low-resource languages, we compare our direct transfer model with the expectation learning model proposed in Zhang et al. (2016). This model is not a direct transfer model, but it does not use any training data in the target languages either. Instead, for each target language, it generates patterns from parallel documents between English and the target language, a large monolingual corpus in the target language, and one-hour interaction with a native speaker of the target language. Note that they also use a crosslingual wikifier, but only for refining the entity types. On the other hand, in our model, the features from the wikifier are used both in detecting entity mention boundaries and entity types. We can see that our approach performs better than their model on all five languages even though we assume much fewer resources. The difference is most significant on Turkish, Tagalog, and Bengali.

\subsection{Quality of Wikifier Features}

One immediate question is, why are wikifier features less helpful on the low-resource languages results than on the CoNLL languages? In this experiment, we show that smaller Wikipedia sizes result in worse Wikipedia features, which is the

\begin{tabular}{lcccc}
\hline \multirow{2}{*}{ FEATURES } & \multicolumn{2}{c}{ SPANISH } & \multicolumn{2}{c}{ GERMAN } \\
& \#inter. & F1 & \#inter. & F1 \\
\hline Wikifier only & $757 \mathrm{~K}$ & 43.82 & $964 \mathrm{~K}$ & 39.83 \\
W.-FB query & $757 \mathrm{~K}$ & 34.69 & $964 \mathrm{~K}$ & 28.27 \\
W.-FB-50\% inter. & $379 \mathrm{~K}$ & 30.32 & $482 \mathrm{~K}$ & 27.24 \\
W.-FB-90\% inter. & $76 \mathrm{~K}$ & 29.44 & $96 \mathrm{~K}$ & 25.94 \\
\hline
\end{tabular}

Table 3: The F1 scores of using only wikifier features with removing the support from FreeBase and varying the number of titles linked to the English Wikipedia. 'W.-FB query' removes the component of querying FreeBase by the target language title from 'Wikifier only' ' $-X \%$ inter.' indicates removing $X \%$ of the interlanguage links with English titles. The column \#inter. shows the number of titles that intersect with English.

reason Yoruba has bad 'Wikifier only' results and then only small improvement from the wikifier features over base features.

The cross-lingual wikifier that we use in our system only grounds words to the intersection of the English and target language Wikipedia. Given a Wikipedia title in the target language, we first retrieve FreeBase IDs by querying the FreeBase API. If it fails, we find the corresponding English Wikipedia title via interlanguage links and then query the API with the English title. However, FreeBase does not contain entities in Yoruba, Bengali, and Tamil, so the first step will always fail for these three languages. We remove this step in the experiments of high-resource languages and the results are shown in the row 'W.-FB query' of Table 3. We see that the performance drops significantly, because many words have no features from FreeBase types.

Next, we randomly remove $50 \%$ and $90 \%$ of the interlanguage links to English titles. This will not only reduce the number of fired features from Wikipedia categories, but also FreeBase types since English titles are used to query FreeBase IDs. When $90 \%$ of interlanguage links are removed, the scores of Spanish and German are closer to Yoruba's score (27.91).

\subsection{Training Languages}

In all previous experiments, the training language is always English. In order to test the efficacy of training with languages other than English, we create a train/test matrix with all combinations of languages, as seen in Figure 2.

The vertical axis represents training language, 


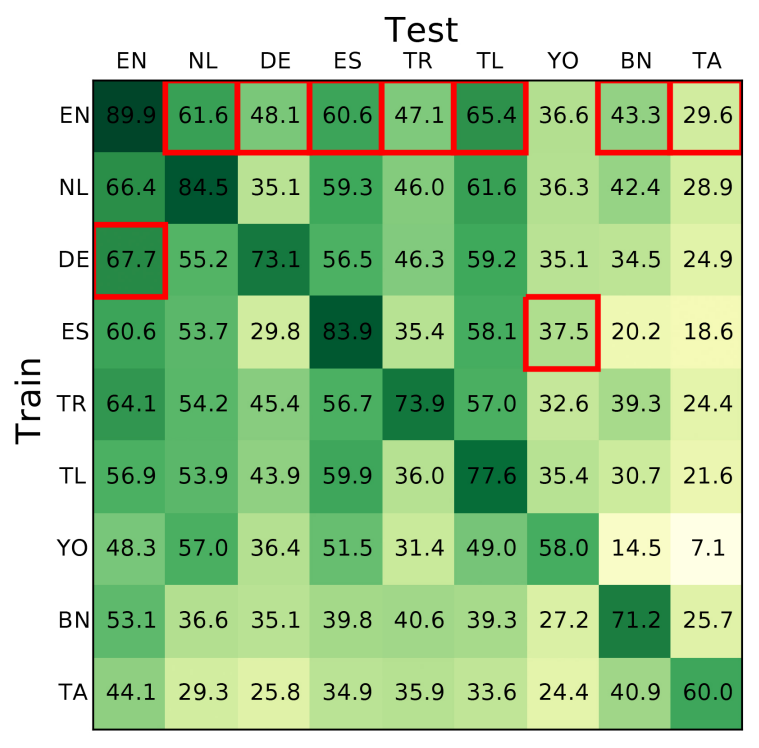

Figure 2: Different training/test language pairs. Scores shown are the F1 scores. The red boxes signify the best non-target training languages.

and the horizontal axis represents test language. A darker color signifies a higher score. For example, if we train on Spanish (ES) and test on Yoruba (YO), we get an F1 of 37.5. When the training or test language is Bengali (BN) or Tamil (TA), we only use wikifier features. For other settings, all features are included. Note that when the test language is one of the CoNLL languages (EN, NL, DE, ES) and the training language is a non-CoNLL language, we ignore all MISC tags in evaluation, since there is no MISC tag in the low-resource languages. The diagonals represent the monolingual setting in which we use all features for all languages. Since we are interested in transferring a model, we ignore the diagonals, and identify the best training language for a given test language as the largest off-diagonal in each column. These are demarcated with red boxes.

English is the best for most languages, with the only exception of Spanish being the best for Yoruba. It makes sense that high-resource languages are better training languages because 1) there are more annotated training instances, 2) larger Wikipedia creates denser wikifier features, therefore providing better estimation of the weights to these features.

Table 4 shows the results of training on multiple languages. We use all features in this experiment. The row "EN" only trains the model on the English training documents, and the results are iden-

\begin{tabular}{lcccc}
\hline TRAINING LANG & TR & TL & YO & AVG \\
\hline EN & 47.12 & 65.44 & 36.65 & 49.74 \\
EN+ES & 44.85 & 66.61 & 37.57 & 49.68 \\
EN+NL & 48.34 & 66.09 & 36.87 & 50.43 \\
EN+DE & 49.47 & 64.10 & 35.14 & 49.57 \\
EN+ES+NL+DE & 49.00 & 66.37 & $\mathbf{3 8 . 0 2}$ & 51.13 \\
ALL-Test Lang & $\mathbf{4 9 . 8 3}$ & $\mathbf{6 7 . 1 2}$ & 37.56 & $\mathbf{5 1 . 5 0}$ \\
\hline
\end{tabular}

Table 4: The F1 scores of the proposed direct transfer model on three low-resource languages using training data in multiple languages. The row "ALL-Test Lang" trains the model on all languages except the test language, Bengali, and Tamil. Bengali and Tamil are excluded since we use all features in this experiment.

tical to those shown in Table 2. Using all CoNLL languages $(\mathrm{EN}+\mathrm{ES}+\mathrm{NL}+\mathrm{DE})$ adds more than 1 point F1 in average comparing to using English only. Finally, training on all but the test languages further improves the results.

This experiment shows that we can augment training data from other languages' annotated documents. Although the performance only increases a little, it does not hurt most of the time.

\subsection{Domain Adaptation}

To improve the results of the monolingual experiments, we consider the domain adaptation setting where there is annotated data for both source and target domains. The question is whether training data from the source domain can improve a model that is trained solely on the target-domain data. In this experiment, we use English as the source domain, and use Spanish, Dutch, Turkish, and Taga$\log$ as four different target domains. We compare three approaches:

- Target: only uses the training data in the target domain. This is the setting of the monolingual experiments in Table 2.

- Src+Tgt: directly uses the training data from both source and target domains. This method is identical to the setting in our previous multi-source direct transfer experiments.

- FrustEasy: the "Frustratingly Easy" adaptation framework proposed by (Daumé, 2007).

All types of features are used in all settings. The results are shown in Table 5. We can see that although $S r c+T g t$ is always the best approach, the improvement over the baseline, Target, is tiny. 


\begin{tabular}{lccccc}
\hline APPROACH & ES & NL & TR & TL & AVG \\
\hline Target & 83.87 & 84.49 & 73.86 & 77.64 & 79.96 \\
Src+Tgt & $\mathbf{8 4 . 1 7}$ & $\mathbf{8 4 . 8 1}$ & $\mathbf{7 4 . 5 2}$ & $\mathbf{7 7 . 8 0}$ & $\mathbf{8 0 . 3 3}$ \\
FrustEasy & 83.89 & 84.08 & 73.73 & 77.04 & 79.69 \\
\hline
\end{tabular}

Table 5: The domain adaptation experiments. The source domain (English) training examples are used to improve the monolingual baseline model (Target) which is only trained on the target domain (Spanish, Dutch, Turkish, and Tagalog) training data. The numbers are the phrase-level F1 scores.

Interestingly, the FrustEasy framework does not help for most languages. This result is consistent with the analysis and observation in Chang et al. (2010) that 1) when the source and target domains are very different, the baseline approach (Target) is very strong, and 2) when there are cross-domain clustering features (e.g., the wikifier features), $S r c+T g t$ is better than FrustEasy. To further improve the monolingual baselines via adaptation from other languages, better crosslingual or language-independent information may be needed.

\section{Conclusion and Discussion}

We propose a language-independent model for cross-lingual NER building on a cross-lingual wikifier. This model works on all languages in Wikipedia and the only requirement is a Wikipedia dump. We study a wide range of languages in both the monolingual and the cross-lingual settings, and show significant improvements over strong baselines. An analysis shows that the quality of the wikifier features depends on the Wikipedia size of the test language.

This work shows that if we can disambiguate words and phrases to the English Wikipedia, the typing information from Wikipedia categories and FreeBase are useful language-independent features for NER. However, there is additional information in Wikipedia that could be helpful and which we do not use, including words in the documents and relations between titles; this would require additional research.

In the future, we would like to experiment with combining our method with other techniques for multilingual NER (Section 2), including parallel projection and the automatic generation of training data from Wikipedia.

\section{Acknowledgments}

This research is supported by NIH grant U54GM114838, a grant from the Allen Institute for Artificial Intelligence (allenai.org), and Contract HR0011-15-2-0025 with the US Defense Advanced Research Projects Agency (DARPA). Approved for Public Release, Distribution Unlimited. The views expressed are those of the authors and do not reflect the official policy or position of the Department of Defense or the U.S. Government.

\section{References}

Rami Al-Rfou, Bryan Perozzi, and Steven Skiena. 2013. Polyglot: Distributed word representations for multilingual NLP. In Proceedings of the Seventeenth Conference on Computational Natural Language Learning, pages 183-192, Sofia, Bulgaria, August. Association for Computational Linguistics.

Rami Al-Rfou, Vivek Kulkarni, Bryan Perozzi, and Steven Skiena. 2015. Polyglot-ner: Massive multilingual named entity recognition. In Proceedings of the 2015 SIAM International Conference on Data Mining, Vancouver, British Columbia, Canada. SIAM.

Ming-Wei Chang, Lev Ratinov, Dan Roth, and Vivek Srikumar. 2008. Importance of semantic represenation: Dataless classification. In Proceedings of the National Conference on Artificial Intelligence (AAAI), July.

Ming-Wei Chang, Michael Connor, and Dan Roth. 2010. The necessity of combining adaptation methods. In Proceedings of the Conference on Empirical Methods for Natural Language Processing (EMNLP), Massachusetts, USA, 10.

D. Das and S. Petrov. 2011. Unsupervised part-ofspeech tagging with bilingual graph-based projections. In Proceedings of the Annual Meeting of the Association for Computational Linguistics (ACL), Portland, OR, June. Association for Computational Linguistics.

Hal Daumé. 2007. Frustratingly easy domain adaptation. In Proceedings of the Annual Meeting of the Association for Computational Linguistics (ACL), pages 256-263, Prague, Czech Republic, June. Association for Computational Linguistics.

Long Duong, Trevor Cohn, Karin Verspoor, Steven Bird, and Paul Cook. 2014. What can we get from 1000 tokens? A case study of multilingual POS tagging for resource-poor languages. In $E M N L P$, pages 886-897. Citeseer.

Maud Ehrmann, Marco Turchi, and Ralf Steinberger. 2011. Building a multilingual named entityannotated corpus using annotation projection. In RANLP. 
Evgeniy Gabrilovich and Shaul Markovitch. 2007. Computing semantic relatedness using Wikipediabased explicit semantic analysis. In IJCAI.

Rebecca Hwa, Philip Resnik, Amy Weinberg, Clara I. Cabezas, and Okan Kolak. 2005. Bootstrapping parsers via syntactic projection across parallel texts. Natural Language Engineering, 11:311-325.

H. Ji, J. Nothman, and B. Hachey. 2015. Overview of tac-kbp2014 entity discovery and linking tasks. In Text Analysis Conference (TAC2014).

H. Ji, J. Nothman, B. Hachey, and R. Florian. 2016. Overview of tac-kbp2015 tri-lingual entity discovery and linking. In Text Analysis Conference (TAC2015).

Jun'ichi Kazama and Kentaro Torisawa. 2007. Exploiting Wikipedia as external knowledge for named entity recognition. In Proceedings of the 2007 Joint Conference of EMNLP-CoNLL, pages 698-707.

Sungchul Kim, Kristina Toutanova, and Hwanjo Yu. 2012. Multilingual named entity recognition using parallel data and metadata from Wikipedia. In $A C L$.

R. McDonald, S. Petrov, and K. Hall. 2011. Multi-source transfer of delexicalized dependency parsers. In Proceedings of the Conference on Empirical Methods for Natural Language Processing (EMNLP), pages 62-72, Edinburgh, Scotland, UK., July. Association for Computational Linguistics.

Andrea Moro, Alessandro Raganato, and Roberto Navigli. 2014. Entity linking meets word sense disambiguation: A unified approach. In Transactions of the Association for Computational Linguistics, volume 2, pages 231-244.

Joel Nothman, Nicky Ringland, Will Radford, Tara Murphy, and James R. Curran. 2012. Learning multilingual named entity recognition from Wikipedia. Artificial Intelligence, 194:151-175.

Lev Ratinov and Dan Roth. 2009. Design challenges and misconceptions in named entity recognition. In Proc. of the Annual Conference on Computational Natural Language Learning (CoNLL), Jun.

Lev Ratinov, Doug Downey, Mike Anderson, and Dan Roth. 2011. Local and global algorithms for disambiguation to Wikipedia. In Proceedings of the Annual Meeting of the Association for Computational Linguistics (ACL).

Avirup Sil and Alexander Yates. 2013. Re-ranking for joint named-entity recognition and linking. In Proceedings of the 22nd ACM international conference on Conference on information \& knowledge management, pages 2369-2374. ACM.

Heather Simpson, Christopher Cieri, Kazuaki Maeda, Kathryn Baker, and Boyan Onyshkevych. 2008. Human language technology resources for less commonly taught languages: Lessons learned toward creation of basic language resources. Collaboration: interoperability between people in the creation of language resources for less-resourced languages, page 7.

Jason R Smith, Chris Quirk, and Kristina Toutanova. 2010. Extracting parallel sentences from comparable corpora using document level alignment. In Human Language Technologies: The 2010 Annual Conference of the North American Chapter of the Association for Computational Linguistics, pages 403-411. Association for Computational Linguistics.

Yangqiu Song and Dan Roth. 2014. On dataless hierarchical text classification. In Proceedings of the $\mathrm{Na}$ tional Conference on Artificial Intelligence (AAAI), 7.

Oscar Täckström, Ryan T. McDonald, and Jakob Uszkoreit. 2012. Cross-lingual word clusters for direct transfer of linguistic structure. In NAACL.

Oscar Täckström. 2012. Nudging the envelope of direct transfer methods for multilingual named entity recognition. In Proceedings of the NAACL-HLT Workshop on the Induction of Linguistic Structure, pages 55-63. Association for Computational Linguistics.

Erik F. Tjong Kim Sang and Fien De Meulder. 2003. Introduction to the conll-2003 shared task: Language-independent named entity recognition. In Walter Daelemans and Miles Osborne, editors, Proceedings of CoNLL-2003, pages 142-147. Edmonton, Canada.

Erik F. Tjong Kim Sang. 2002. Introduction to the CoNLL-2002 shared task: Language-independent named entity recognition. In Proceedings of CoNLL-2002, pages 155-158. Taipei, Taiwan.

Chen-Tse Tsai and Dan Roth. 2016. Cross-lingual wikification using multilingual embeddings. In NAACL.

Mengqiu Wang and Christopher D Manning. 2014. Cross-lingual projected expectation regularization for weakly supervised learning. In $T A C L$.

Fei Wu and Daniel S. Weld. 2010. Open information extraction using Wikipedia. In $A C L$.

David Yarowsky, Grace Ngai, and Richard Wicentowski. 2001. Inducing multilingual text analysis tools via robust projection across aligned corpora. In Proceedings of the first international conference on Human language technology research, pages 18. Association for Computational Linguistics.

Boliang Zhang, Xiaoman Pan, Tianlu Wang, Ashish Vaswani, Heng Ji, Kevin Knight, and Daniel Marcu. 2016. Name tagging for low-resource incident languages based on expectation-driven learning. In NAACL. 